\title{
Poster: Symbolic Control of Monotone Systems Application to Ventilation Regulation in Buildings *
}

\author{
Pierre-Jean Meyer \\ LJK \& GIPSA-lab \\ University of Grenoble, France \\ Pierre-Jean.Meyer@imag.fr
}

\author{
Antoine Girard \\ LJK \\ University of Grenoble, France \\ Antoine.Girard@imag.fr
}

\author{
Emmanuel Witrant \\ GIPSA-lab \\ University of Grenoble, France \\ Emmanuel.Witrant@ujf-grenoble.fr
}

\begin{abstract}
We describe an application of symbolic control to ventilation regulation in buildings. The monotonicity property of a nonlinear control system subject to disturbances, modeling the process, is exploited to obtain symbolic abstractions, in the sense of alternating simulation. The resulting abstractions consist of non-deterministic finite transition systems, for which we can synthesize supervisory safety controllers to keep the room temperatures within prescribed bounds. To choose among possible control inputs preserving safety, we consider the problem of minimizing a given cost function and apply a receding horizon control scheme. The approach has been applied to temperature regulation on a small-scale building equipped with underfloor air distribution (UFAD). To the best of our knowledge, this is the first report of experimental implementation of symbolic controllers.
\end{abstract}

\section{Categories and Subject Descriptors}

I.2.8 [Artificial Intelligence]: Problem Solving, Control Methods, and Search - Control theory; J.7 [Computer Applications]: Computers in other systems-Command and control

\section{Keywords}

Symbolic control; Monotone system; Application.

\section{SYMBOLIC ABSTRACTION}

We consider a nonlinear control system of the form

$$
\dot{x}=f(x, u, w) \text { with } x \in \mathbb{R}^{n}, u \in \mathbb{R}^{p} \text { and } w \in \mathbb{R}^{q}
$$

where $x$ denotes the state, $u$ the control input and $w$ the disturbance input. We assume that the control and disturbance inputs are bounded in multidimensional intervals: $u \in[\underline{u}, \bar{u}]$

\footnotetext{
* This work was partly supported by a PhD scholarship and the research project COHYBA funded by Région RhôneAlpes.
}

and $w \in[w, \bar{w}]$. The trajectories of the system are denoted $\Phi\left(\cdot, x_{0}, \mathbf{u}, \mathbf{w}\right)$ where $\Phi\left(t, x_{0}, \mathbf{u}, \mathbf{w}\right)$ is the state reached at time $t \in \mathbb{R}_{0}^{+}$from initial state $x_{0} \in \mathbb{R}^{n}$, under piecewise continuous control and disturbance inputs $\mathbf{u}: \mathbb{R}_{0}^{+} \rightarrow \mathbb{R}^{p}$ and $\mathbf{w}: \mathbb{R}_{0}^{+} \rightarrow \mathbb{R}^{q}$. We also assume that the system is cooperative, which is a subclass of monotone systems [1].

Definition 1 (Cooperative System). System (1) is cooperative if for all $x \geq x^{\prime}, \mathbf{u} \geq \mathbf{u}^{\prime}, \mathbf{w} \geq \mathbf{w}^{\prime}$, it holds for all $t \geq 0, \Phi(t, x, \mathbf{u}, \mathbf{w}) \geq \Phi\left(t, x^{\prime}, \mathbf{u}^{\prime}, \mathbf{w}^{\prime}\right)$, where $\geq$ denotes the componentwise inequality.

We describe the dynamics of the sampled version of system (1) with time period $\tau$ as a non-deterministic transition system $S$ as presented in [4]. The control objective is to keep the state in an interval $[\underline{x}, \bar{x}]$.

We define a symbolic abstraction of $S$ as a finite transition system whose states are the elements of a partition of $\mathbb{R}^{n}$, $\mathcal{P}^{*}=\mathcal{P} \cup\{$ Out $\}$ where $\mathcal{P}$ is a partition of $[\underline{x}, \bar{x}]$ into intervals. The abstraction is $S_{a}=\left(X_{a}, X_{a 0}, U_{a}, \longrightarrow\right)$ where the set of states $X_{a}=\mathcal{P}^{*}$, the set of initial states $X_{a 0}=\mathcal{P}$, the set of inputs $U_{a}$ is a discretization of $[\underline{u}, \bar{u}]$, and the transition relation is given for all $s=[\underline{s}, \bar{s}] \in \mathcal{P}, s^{\prime} \in \mathcal{P}^{*}, u \in U_{a}$ by:

$$
s \stackrel{u}{\longrightarrow} s^{\prime} \Longleftrightarrow s^{\prime} \cap[\Phi(\tau, \underline{s}, u, \underline{w}), \Phi(\tau, \bar{s}, u, \bar{w})] \neq \emptyset .
$$

As we deal with transition systems with control inputs and non-determinism, we are interested in alternating simulation relations as behavioral relationships between $S$ and $S_{a}$ [4]. The cooperativeness assumption allows us to prove the following result.

Proposition 1. The symbolic abstraction $S_{a}$ is alternatingly simulated by the original transition system $S$.

As a consequence, if we design a safety controller for $S_{a}$ keeping its state in $\mathcal{P}$, the alternating simulation relation provides an equivalent safety controller for $S$ keeping its state in $[\underline{x}, \bar{x}]$.

\section{SYMBOLIC CONTROL}

Using a classical fixed-point algorithm [5], we can synthesize a supervisory safety controller $C: \mathcal{P} \rightarrow 2^{U_{a}}$ for $S_{a}$ keeping its state in $\mathcal{P}$.

To choose among possible control inputs preserving safety, we consider the cost function $J_{0}$ defined iteratively by:

$$
\begin{gathered}
J_{N}(s)=\hat{g}(s) \\
J_{k}(s)=\min _{u \in C(s)}\left(g(s, u)+\lambda \max _{s \stackrel{u}{\longrightarrow} s^{\prime}} J_{k+1}\left(s^{\prime}\right)\right)
\end{gathered}
$$



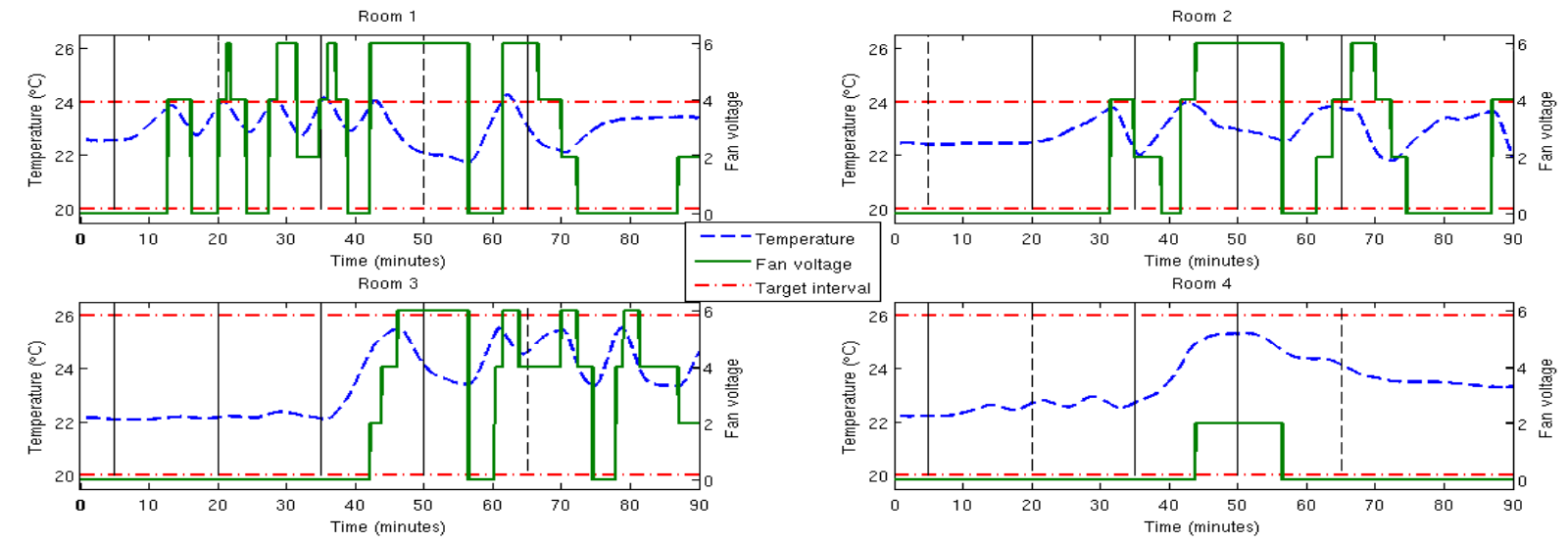

Figure 1: UFAD experiment controlled with a symbolic method.

where $N \in \mathbb{N}$ is the time horizon, $\lambda \in(0,1)$ is a discount factor, $\hat{g}: \mathcal{P} \rightarrow \mathbb{R}^{+}$and $g: \mathcal{P} \times U_{a} \rightarrow \mathbb{R}^{+}$are cost functions. Then, we apply a receding horizon control scheme given by the controller for $S_{a}$ :

$$
C_{a}^{*}(s)=\underset{u \in C(s)}{\arg \min }\left(g(s, u)+\lambda \max _{s \stackrel{u}{\longrightarrow} s^{\prime}} J_{1}\left(s^{\prime}\right)\right) .
$$

For the original transition system $S$, we define the associated controller $C^{*}$ given for all $s \in \mathcal{P}, x \in s$, by $C^{*}(x)=C_{a}^{*}(s)$. Note that all the above computations required to obtain $C^{*}$ (abstraction and controller synthesis) can be done offline. We can also prove the following result showing that $C^{*}$ ensures safety of $S$ with performance guarantees.

Proposition 2. Let $\left(x_{0}, u_{0}, x_{1}, u_{1}, \ldots\right)$ be a trajectory of $S$ controlled with $C^{*}$, then for all $k \in \mathbb{N}, x_{k} \in[\underline{x}, \bar{x}]$. Moreover, let $s_{0}, s_{1}, \cdots \in \mathcal{P}$ such that for all $k \in \mathbb{N}, x_{k} \in s_{k}$. Then, it holds for all $k \in \mathbb{N}$,

$$
\sum_{i=0}^{+\infty} \lambda^{i} g\left(s_{k+i}, u_{k+i}\right) \leq J_{0}\left(s_{k}\right)+\frac{\lambda^{N+1}}{1-\lambda} M
$$

where $M$ is an upper bound of functions $g$ and $\hat{g}$.

\section{UNDERFLOOR AIR DISTRIBUTION}

The UnderFloor Air Distribution (UFAD) is an alternative solution to traditional ceiling based ventilation in buildings, where the air is cooled down in an underfloor plenum and then sent into each room when needed. The system considered is based on a 4-room small-scale experimental building equipped with UFAD sketched in Figure 2. A model of the temperature variations in each room is derived from the energy and mass conservation equations in the room [2]. The obtained model is an ordinary differential equation involving the temperature of each room (the state), the ventilation from the underfloor (control input in each room) and continuous and discrete disturbances (outside temperature, door opening, ... ) This model is proven to be cooperative [2] and validated by an identification procedure on the building [3].

The symbolic control method is applied to this model and the resulting control strategy is implemented in the 4-room experimental building. In Figure 1 are displayed the measured temperatures (dashed blue, on the left axis) and the controlled ventilation (plain green, on the right axis) dis-

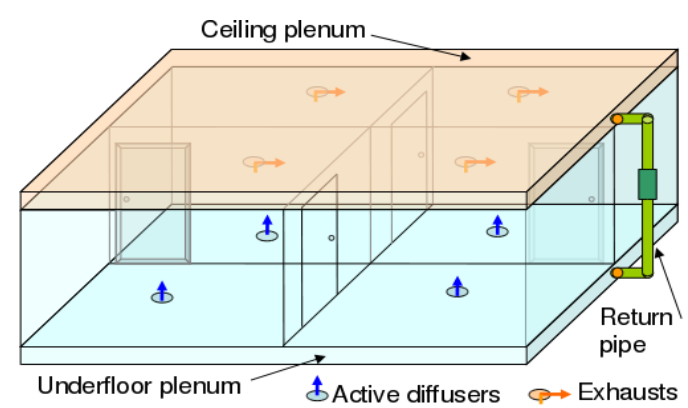

Figure 2: 4-room flat equipped with UFAD.

cretized into 256 values. The prescribed bounds on the temperature are represented by dash-dotted horizontal lines on the figure. The symbolic abstraction was computed on a partition consisting of $10^{4}$ intervals. The performance criterion specifies the desired tradeoff between the magnitude of the control inputs, their variations and the distance of the state to the center of the interval given by the temperature bounds, with a time horizon $N=5$ and discount factor $\lambda=0.5$. We can see that the safety specification is met: the temperatures are maintained within the prescribed bounds despite the effect of external disturbances.

\section{REFERENCES}

[1] D. Angeli and E. D. Sontag. Monotone control systems. IEEE Transactions on Automatic Control, 48(10):1684-1698, 2003.

[2] P.-J. Meyer, A. Girard, and E. Witrant. Controllability and invariance of monotone systems for robust ventilation automation in buildings. In Proc. of the $52^{\text {nd }}$ IEEE Conference on Decision and Control, 2013.

[3] P.-J. Meyer, H. Nazarpour, A. Girard, and E. Witrant. Experimental implementation of UFAD regulation based on robust controlled invariance. In Proc. of the $13^{\text {th }}$ European Control Conference, 2014.

[4] P. Tabuada. Verification and control of hybrid systems: a symbolic approach. Springer, 2009.

[5] W. M. Wonham and P. J. Ramadge. On the supremal controllable sublanguage of a given language. SIAM Journal on Control and Optimization, 25(3):637-659, 1987. 